\title{
ANALYSIS OF CLOCK JITTER ERROR IN MULTIBIT CONTINUOUS-TIME $\Sigma \Delta$ MODULATORS WITH NRZ FEEDBACK WAVEFORM
}

\author{
Ramón Tortosa, José M. de la Rosa, Angel Rodríguez-Vázquez and Francisco V. Fernández \\ Instituto de Microelectrónica de Sevilla - IMSE-CNM (CSIC) \\ Edificio CICA-CNM, Avda. Reina Mercedes s/n, 41012- Sevilla, SPAIN \\ Phone: +34 95 5056666, Fax: +34 95 5056686, E-mail: \{tortosa|jrosa|angel|pacov\}@imse.cnm.es
}

\begin{abstract}
This paper presents a detailed study of the clock jitter error in multibit continuous-time $\Sigma \Delta$ modulators with non-return-to-zero feedback waveform. Closed-form expressions are derived for the in-band error power and the signal-to-noise ratio showing that the jitter-induced noise can be separated into two main components: one depending on the modulator loop filter and the other one due to the input signal. The latter, not considered in previous approaches, allows us to accurately predict the signal-to-noise ratio degradation and to optimize the modulator performance in terms of jitter insensitivity. Moreover, the use of state-space formulation makes the analysis quite general and applicable to either cascaded or single-loop architectures. Time-domain simulations of several modulators are shown to validate the presented approach. ${ }^{\dagger 1}$
\end{abstract}

\section{INTRODUCTION}

Nowadays, the increasing demand for ever faster Analog-to-Digital Converters (ADCs) in broadband communication systems has boosted the interest in Continuous-Time (CT) $\underline{\text { Sigma-Delta }}$ Modulators $(\Sigma \Delta \mathrm{Ms})$. These modulators offer an intrinsic antialiasing filtering and provide potentially higher sampling rates with lower power consumption than their Discrete-Time (DT) counterparts [1][2]. However, CT $\Sigma \Delta \mathrm{Ms}$ are more sensitive than DT $\Sigma \Delta \mathrm{Ms}$ to several circuit non idealities. One of their major degrading factors, especially in high-speed applications, is due to uncertainties in the clock signal edges, commonly referred to as clock jitter [1].

Clock jitter in CT $\Sigma \Delta \mathrm{Ms}$ has been object of several studies reported in open literature [1][3]-[7]. Most of them were carried out considering $\Sigma \Delta \mathrm{M}$ architectures with an internal single-bit quantiz-

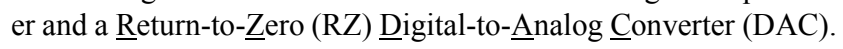
However, multibit quantization has been used in most silicon prototypes achieving medium-high resolutions (11-14 bit) within high signal bandwidths (1-15 MHz) [8]-[10]. The combined use of high-order (3rd-4th order) single-loop architectures with multibit (3-6 bit) quantization allows to reduce the oversampling ratio (normally $<12$ ) while guaranteeing stability and robustness with respect to circuit parameter tolerances - the latter being a very critical error in CT $\Sigma \Delta \mathrm{Ms}$ [1][2]. In addition to improve resolution, multibit quantization can reduce the sensitivity of CT $\Sigma \Delta \mathrm{Ms}$ to clock jitter if a Non-Return-to-Zero (NRZ) feedback waveform is used in the DAC [9]. Therefore, their study is needed in order to optimize the modulator performance in terms of sensitivity to jitter error.

The analysis of clock jitter in CT $\Sigma \Delta$ Ms considering a NRZ feedback waveform is mathematically more complex than using a RZ

$\dagger 1$. This work has been supported by the Spanish Ministry of Science and Education (with support from the European Regional Development Fund) under contract TEC2004-01752/MIC. pulse shaping. In most cases, designers resort to semi-empirical estimations based on simulation results and consider a white-noise model for the jitter error [1][5][9]. To the best of the authors' knowledge, only the work in [7] takes into account the effect of the modulator loop filter transfer function on the in-band jitter noise power of CT $\Sigma \Delta \mathrm{Ms}$ with NRZ DAC. However, the analysis in [7] does not consider the effect of input signal for the sake of simplicity.

This paper analyzes the effect of signal-dependent clock jitter in multibit CT $\Sigma \Delta$ Ms with NRZ DAC. State-space formulation [11] is used to derive closed-form relations among jitter error, modulator specifications, loop filter transfer function and input signal parameters. The results of this study - applicable to any modulator topology - show effects not considered in previous approaches which become critical in high frequency applications. As an illustration several modulators using either single-loop or cascaded topologies are simulated to demonstrate the theoretical predictions.

\section{CLOCK JITTER IN CT $\Sigma \Delta M S$ WITH NRZ DACS}

Fig.1 shows the conceptual block diagram of a single-loop CT $\Sigma \Delta \mathrm{M}$. The loop filter is $\mathrm{CT}$ and the sampling operation is realized before quantization instead of at the modulator input as done in the case of DT $\Sigma \Delta \mathrm{Ms}$. Thus, the output signal, $y(n)^{\dagger 2}$, is DT, the input signal, $x(t)$, is CT and a DT-to-CT transformation is implemented by the DAC to create the CT feedback signal, $y(t)$. Therefore, there are two clocked building blocks subject to jitter error: the sampler and the DAC. The error introduced through the sampling process is reduced by the loop gain and shaped in the same way as the quantization noise and hence, its effect can be neglected. On the contrary, the jitter error associated to the DAC directly adds with the input signal, thus increasing the in-band noise power and degrading the modulator performance.

In the case of a NRZ DAC, the error sequence can be related to the output signal using the following relationship [1]:

$$
\varepsilon(n)=(y(n)-y(n-1)) \frac{\Delta T(n)}{T_{s}}
$$

where $T_{S}$ is the sampling period and $\Delta T(n)$ is the time uncertainty.

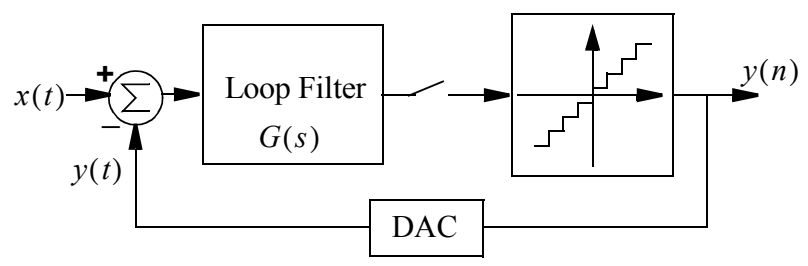

Figure 1. Conceptual block diagram of a single-loop CT $\Sigma \Delta \mathrm{M}$.

$\dagger 2$. In order to simplify the notation, $y\left(n T_{s}\right)$ is written as $y(n)$ with $T_{s}$ being the sampling period. 
Assuming that the input signal and the quantization error are uncorrelated and that $\Delta T(n)$ is a Gaussian random process with zero mean and standard deviation $\sigma_{\Delta T}$, the power of the jitter error signal can be written as:

$$
\begin{aligned}
& P_{\varepsilon}=E\left\{\varepsilon(n)^{2}\right\}=\frac{\sigma^{2} \Delta T}{T_{s}^{2}} E\left\{[y(n)-y(n-1)]^{2}\right\} \cong \\
& \cong \frac{\sigma_{\Delta T}^{2}}{T_{s}^{2}}\left(E\left\{(x(n)-x(n-1))^{2}\right\}+E\left\{(q(n)-q(n-1))^{2}\right\}\right)
\end{aligned}
$$

where $E\{$.$\} stands for the mathematical expectation [12] and$ $q(n)$ is the shaped quantization noise, given by:

$$
q(z)=N_{T F}(z) e(z)
$$

where $N_{T F}(z)$ represents the quantization Noise $\underline{\text { Transfer }}$ Function and $e(z)$ is the quantization error - assumed to be a white noise source.

Considering a sinewave input signal of amplitude $A$ and angular frequency $\omega_{i}=2 \pi f_{i}, \Delta x_{n} \equiv[x(n)-x(n-1)]$ can be simplified as:

$$
\left.\Delta x_{n} \cong \frac{\mathrm{d}}{\mathrm{d} t} x(t)\right|_{t=n T_{s}} \cdot T_{s}=A \omega_{i} T_{s} \cos \left(\omega_{i}(n-1) T_{s}\right)
$$

and hence,

$$
E\left\{\left(\Delta x_{n}\right)^{2}\right\}=T_{s}^{2} A^{2} \omega_{i}^{2} E\left\{\left(\cos \left(\omega_{i}(n-1) T_{s}\right)\right)^{2}\right\}=\frac{T_{s}^{2} A^{2} \omega_{i}^{2}}{2}(5)
$$

The expectation value of $\Delta q_{n} \equiv[q(n)-q(n-1)]$ can be derived from (3) giving:

$$
\begin{gathered}
E\left\{\left(\Delta q_{n}\right)^{2}\right\}=E\left\{\left(Z^{-1}\left[\left(1-z^{-1}\right) N_{T F}(z) e(z)\right]\right)^{2}\right\}= \\
=\frac{X_{F S}^{2}}{12 \pi\left(2^{B}-1\right)^{2}} \int_{0}^{\pi}\left|\left(1-e^{-j \omega}\right) N_{T F}\left(e^{-j \omega}\right)\right|^{2} d \omega
\end{gathered}
$$

where $X_{F S}$ and $B$ are the full-scale and the internal number of bits of the quantizer, respectively.

From (2), (5) and (6), we obtain:

$P_{\varepsilon} \cong\left(\frac{\sigma_{\Delta T}}{T_{s}}\right)^{2} \cdot\left(\frac{A^{2} \omega_{i}^{2}}{2 f_{s}^{2}}+\frac{X_{F S}^{2}}{12 \pi\left(2^{B}-1\right)^{2}} \int_{0}^{\pi}\left|\left(1-e^{-j \omega}\right) N_{T F}\left(e^{-j \omega}\right)\right|^{2} d \omega\right)$

where $f_{s} \equiv 1 / T_{s}$ is the sampling frequency.

In some modulator topologies, the integration in (7) may become mathematically too complex, thus requiring the use of numerical solving methods. This can be simplified if the state-space formulation is used to derive $E\left\{\left(\Delta q_{n}\right)^{2}\right\}$ as shown in next section.

\section{STATE-SPACE FORMULATION}

Fig. 2 shows the state-space representation of $N_{T F}(z)$, which can be described by the following finite difference equations [12]:

$$
\begin{gathered}
\overline{v(n+1)_{0}}=\overline{F_{0}} \cdot \overline{v(n)_{0}}+\overline{p_{0}} \cdot e(n) \\
q(n)={\overline{g_{0}}}^{T} \cdot \overline{v(n)_{0}}+e(n)
\end{gathered}
$$

where $\bar{F}_{0}$ is the state matrix, $\overline{v(n)_{0}}$ is the $L \times 1$ state vector, $\overline{p_{0}}$ and $\overline{g_{0}}$ are $L \times 1$ vectors and $L$ is the order of $N_{T F}$.

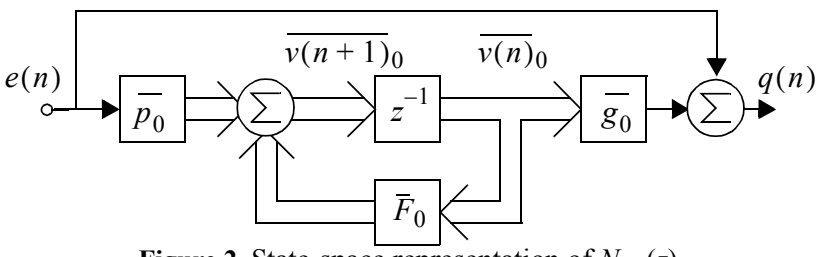

Figure 2. State-space representation of $N_{T F}(z)$.

Equation system (8) can be solved recursively to find the relation between the initial state $\left(\overline{v\left(n_{0}\right)_{0}}\right)$, previous input $(e(k))$, present input $(e(n))$ and output $(q(n))$ of the system [12]. This gives:

$$
\begin{aligned}
& q(n)={\overline{g_{0}}}^{T} \cdot{\overline{F_{0}}}^{n-n_{0}} \cdot \overline{v\left(n_{0}\right)_{0}}+ \\
& +\sum_{k=0}^{n-1}{\overline{g_{0}}}^{T} \cdot{\overline{F_{0}}}^{n-1-k} \cdot \overline{p_{0}} \cdot e(k)+e(n)
\end{aligned}
$$

Assuming that the initial state, $\overline{v\left(n_{0}\right)}$, is zero and considering that $E\{e(k) e(j)\}=0$ for $k \neq j$, it can be shown from (9) that:

$$
E\left\{q^{2}(n)\right\}=E\left\{e^{2}(n)\right\}\left(1+\sum_{k=0}^{n-1}\left[{\overline{g_{0}}}^{T} \cdot \bar{F}_{0}^{n-1-k} \cdot \bar{p}_{0}\right]^{2}\right)
$$

Diagonalizing $\bar{F}_{0}$ and considering that the system in Fig.2 is stable, the expression in (10) can be re-written as:

$$
E\left\{q(n)^{2}\right\}=E\left\{e(n)^{2}\right\}\left(1-\sum_{k=1 j=1}^{L} \sum_{k} g_{k} p_{k} g_{j} p_{j} \frac{\lambda_{k}^{-1} \lambda_{j}^{-1}}{1-\lambda_{k}^{-1} \lambda_{j}^{-1}}\right)
$$

where $\lambda_{i}$ are the eigenvalues of $\overline{F_{0}}$ and $g_{i}$ and $p_{i}$ are respectively the elements of $\bar{g}=\bar{g}_{0} \cdot \bar{T} \quad$ and $\bar{p}=T^{-1} \cdot \overline{p_{0}}$, with $\bar{T}$ being the matrix of the eigenvectors of $\bar{F}_{0}$.

Using a similar procedure, it can be demonstrated that:

$$
\begin{gathered}
E\{q(n) q(n-1)\}= \\
=E\left\{e(n)^{2}\right\}\left(\frac{-T}{g} \cdot \bar{p}-\sum_{k=1} \sum_{j=1} g_{k} p_{k} g_{j} p_{j} \frac{\lambda_{k}^{-1}}{1-\lambda_{k}^{-1} \lambda_{j}^{-1}}\right)
\end{gathered}
$$

Taking into account that $E\left\{e(n)^{2}\right\}=X_{F S}^{2} /\left[12 \cdot\left(2^{B}-1\right)^{2}\right]$, and $E\left\{(q(n))^{2}\right\}=E\left\{(q(n-1))^{2}\right\}$, the value of $E\left\{\left(\Delta q_{n}\right)^{2}\right\}$ can be derived from (11) and (12) as:

$$
\begin{aligned}
E\left\{\left(\Delta q_{n}\right)^{2}\right\}= & 2\left[E\left\{(q(n))^{2}\right\}-(E\{q(n) q(n-1)\})\right]= \\
= & \frac{X_{F S}^{2}}{6\left(2^{B}-1\right)^{2}} \cdot \psi(\bar{g}, \bar{p}, \bar{\lambda}, L)
\end{aligned}
$$

where

$$
\psi(\bar{g}, \bar{p}, \bar{\lambda}, L)=1-\bar{g}^{-T} \cdot \bar{p}+\sum_{k=1 j=1}^{L} \sum_{k} g_{k} p_{k} g_{j} p_{j} \frac{\lambda_{k}^{-1}-\lambda_{k}^{-1} \lambda_{j}^{-1}}{1-\lambda_{k}^{-1} \lambda_{j}^{-1}}
$$

Replacing (6) with (13) in (7) and assuming that the jitter noise is an additive noise source at the input of the modulator, the Signal-to-№ise $\underline{\text { Ratio }}(S N R)$ dominated by jitter can be written as:

$$
S N R=\frac{A^{2}}{B_{w} \cdot\left(\sigma_{\Delta T}\right)^{2} \cdot\left[\frac{A^{2} \omega_{i}^{2}}{f_{s}}+\frac{X_{F S}^{2} \cdot f_{s}}{3\left(2^{B}-1\right)^{2}} \psi(\bar{g}, \bar{p}, \bar{\lambda}, L)\right]}
$$


where $B_{w}$ is the signal bandwidth.

Note that in the denominator of $(15),\left(\sigma_{\Delta T}\right)^{2}$ is multiplied by a factor that is the sum of two terms: one depending on the input signal parameters $\left(A\right.$ and $\left.\omega_{i}\right)$ and the other one which is a function of the modulator topology parameters ( $B, X_{F S}$, $\psi(g, p, \lambda, L))$. The first term decreases with $f_{s}$ while the second term increases with $f_{s}$. This is illustrated in Fig. 3 where the two terms in brackets in the denominator of (15) are plotted versus $f_{s}$ for a 5-bit 3rd-order single-loop CT $\Sigma \Delta \mathrm{M}$ with $f_{i}=B_{w}=20 \mathrm{MHz}$. Note that there is an optimum value of $f_{s}$, $f_{\text {sop }}=170 \mathrm{MHz}$, that minimizes the in-band jitter noise power and hence, maximizes $S N R^{\dagger 3}$. Thus, using (15) as a figure of merit in multibit CT $\Sigma \Delta$ Ms with NRZ DAC in which jitter is the main limiting factor, the modulator performance can be optimized for given specifications in terms of loop filter parameters, sampling frequency and the number of bits of the internal quantizer.

It is important to mention that the effect of $N_{T F}$ on the in-band jitter noise of multibit NRZ CT $\Sigma \Delta \mathrm{Ms}$ was previously studied in [7]. However, it did not take into account the impact of signal-dependent jitter term, which can be very critical in broadband applications as illustrated in next section.

\section{SIMULATION RESULTS}

The presented study has been validated through time-domain behavioural simulation using SIMSIDES, a SIMULINK-based simulator for $\Sigma \Delta \mathrm{Ms}$ [13]. Fig. 4 shows the multibit NRZ CT $\Sigma \Delta$ Ms under study. Fig.4(a) is a 3rd-order single-loop and Fig.4(b) is a cascaded 2-1 topology. In both architectures, feed-forward stabilization is used and a feedback coefficient $k_{r}$ is used to move one of the poles to an optimum position [14]. The modulators were synthesized to handle signals within $B_{w}=20 \mathrm{MHz}$ for VDSL application.

Three different cases are considered:

- CT $\Sigma \Delta$ M1: Fig.4(a) with $f_{s}=400 \mathrm{MHz}$ and $B=2$

- CT $\Sigma \Delta \mathrm{M} 2$ : Fig.4(a) with $f_{s}=160 \mathrm{MHz}$ and $B=5$

- CT $\Sigma \Delta$ M3: Fig.4(b) with $f_{s}=160 \mathrm{MHz}$ and $B_{1}=B_{2}=5$

where $B_{1}$ and $B_{2}$ are respectively the number of bits of the quantizer in the first- and second- stage in Fig.4(b). Table 1 shows the values of the loop-filter coefficients $\left(k_{i}\right)$ as well as the position of the poles and Table 2 shows the values of $g_{i}, p_{i}$ and $\lambda_{i}$ for the three cases mentioned above.

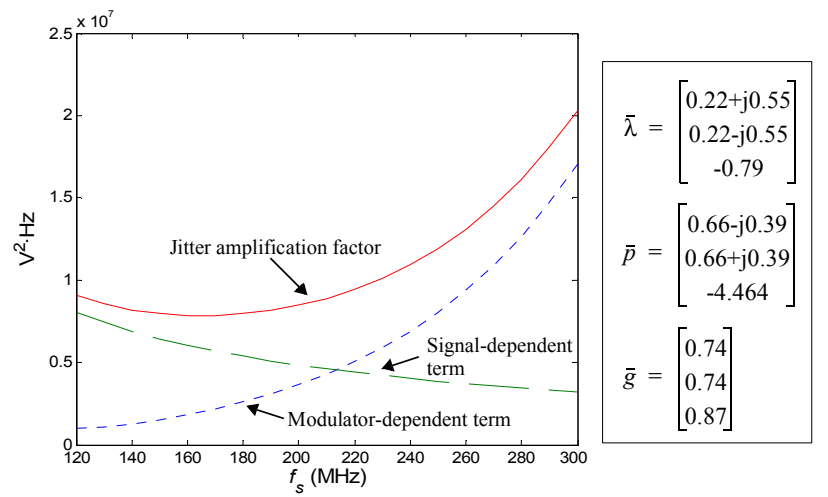

Figure 3. Jitter components vs. $f_{s}$ for a 5-bit 3rd order CT $\Sigma \Delta \mathrm{M}$.

$\left\lceil 3\right.$. The values of $g_{i}, p_{i}$ and $\lambda_{i}$ for $f_{s}=f_{\text {sop }}=170 \mathrm{MHz}$ are shown in Fig.3.

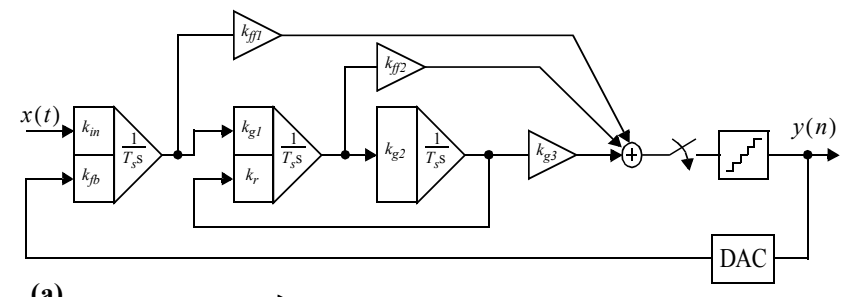

(a)

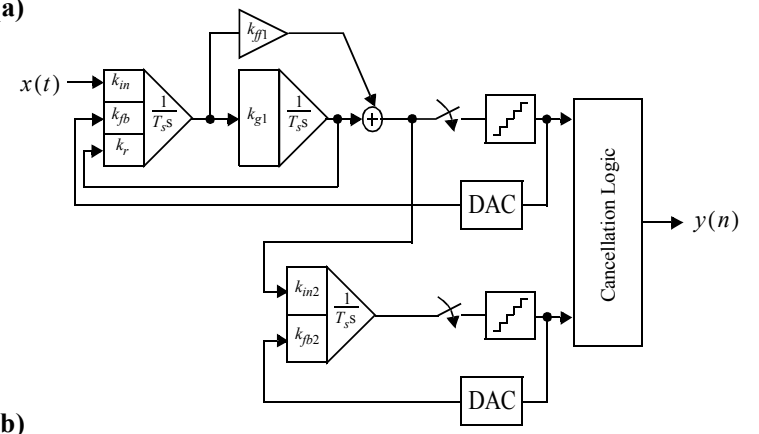

Figure 4. Multibit NRZ CT $\Sigma \Delta$ Ms under study. (a) 3rd-order single-loop architecture. (b) Cascaded 2-1 architecture.

Fig. 5 shows several simulated output spectra of cases CT $\Sigma \Delta \mathrm{M} 1$ (Fig.5(a)) and CT $\Sigma \Delta \mathrm{M} 2$ (Fig.5(b)) corresponding to different values of $f_{i}$ and $\sigma_{\Delta T}=25 \mathrm{ps}$. Note that in Fig.5(a), the in-band noise power does not depend on $f_{i}$ as predicted by [7]. However, as $B$ increases from $B=2$ to $B=5$, the modulator-dependent term in (15) decreases and hence, the in-band noise is dominated by the signal-dependent term as illustrated in Fig.5(b). This effect is better shown in Fig.6, where the $S N R$-peak of the modulators in Fig.4 is plotted vs. $\sigma_{\Delta T}$ for several values of $f_{i}$, showing simulation results and theoretical predictions ${ }^{\dagger 4}$. For comparison pur-

Table 1: Loop-filter coefficients of CT $\Sigma \Delta$ Ms in Fig.4

\begin{tabular}{|c|c|c|c|}
\cline { 2 - 4 } \multicolumn{1}{c|}{} & CT $\Sigma \Delta \mathrm{M} 1$ & CT $\Sigma \Delta \mathrm{M} 2$ & CT $\Sigma \Delta \mathrm{M} 3$ \\
\hline \hline$k_{i n}$ & 1.5 & 2 & 1.6 \\
\hline$k_{f b}$ & -1.5 & -2 & -1.6 \\
\hline$k_{g 1}$ & 1 & 1 & 1.6 \\
\hline$k_{r}$ & -0.1 & -0.37 & -0.24 \\
\hline$k_{g 2}$ & 0.6 & 1 & - \\
\hline$k_{g 3}$ & 0.5 & 1.2 & - \\
\hline$k_{f f 1}$ & 1 & 1 & 1 \\
\hline$k_{f f 2}$ & 0.5 & 1 & - \\
\hline$k_{i n 2}$ & - & - & 1 \\
\hline$k_{f b 2}$ & - & - & -1 \\
\hline Poles & \multicolumn{2}{|c|}{$\omega_{1}=0$} & $\omega_{2}=\sqrt{3 / 5} \cdot 2 \pi \cdot B_{w}$ \\
\hline
\end{tabular}

Table 2: Values of $g_{i}, p_{i}$ and $\lambda_{i}$ for the CT $\Sigma \Delta$ Ms in Fig.4

\begin{tabular}{|c|c|c|c|}
\cline { 2 - 4 } \multicolumn{1}{c|}{} & $\mathrm{CT} \Sigma \Delta \mathrm{M} 1$ & $\mathrm{CT} \Sigma \Delta \mathrm{M} 2$ & $\mathrm{CT} \Sigma \Delta \mathrm{M} 3$ \\
\hline \hline $\bar{\lambda}$ & {$[-0.328$} & {$[0.122+\mathrm{j} 0.39$} & {$[-0.543+\mathrm{j} 0.64$} \\
& $0.664-\mathrm{j} 0.438$ & $0.122-\mathrm{j} 0.39$ & $-0.543-\mathrm{j} 0.64]$ \\
& $0.664-\mathrm{j} 0.438]$ & $-0.667]$ & \\
\hline $\bar{g}$ & {$[-0.560 .8530 .853]$} & {$[0.810 .810 .959]$} & {$[0.7660 .766]$} \\
\hline $\bar{p}$ & {$[3.503$} & {$[1.476+\mathrm{j} 1.722 \mathrm{E}-3$} & {$[-1.78-\mathrm{j} 1.81$} \\
& $0.016-\mathrm{j} 0.184$ & $1.476-\mathrm{j} 1.722 \mathrm{E}-3$ & $-1.78+\mathrm{j} 1.81]$ \\
& $0.016+\mathrm{j} 0.184]$ & $-5.69]$ & \\
\hline
\end{tabular}




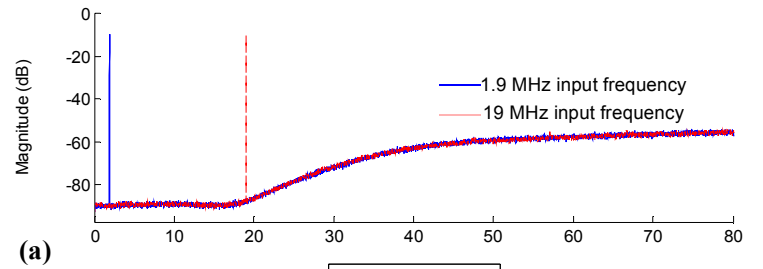

(a)

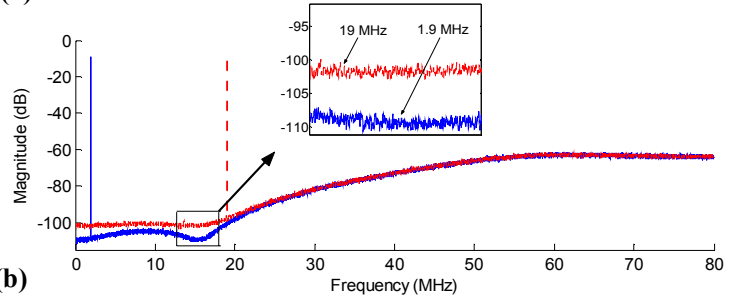

(b)

Figure 5. Effect of jitter error on the output spectra of (a) CT $\Sigma \Delta \mathrm{M} 1$ and (b) CT $\Sigma \Delta \mathrm{M} 2$.

poses, predictions given by [7] are also included. Note that, simulated and theoretical data matched very well when the combined effect of signal- and modulator-dependent jitter noise is taken into account as shown in this work.

\section{CONCLUSIONS}

The effect of clock jitter error on multibit CT $\Sigma \Delta \mathrm{Ms}$ with NRZ $\mathrm{DAC}$ has been analyzed. Based on the use of state-space formulation, easy-to-compute closed-form expressions have been derived for the noise power and signal-to-noise ratio. It has been demonstrated that the jitter-induced noise has two components: one depending on signal parameters and the other one depending on the modulator loop filter. Their combined effect, not predicted by previous approaches, has been confirmed by time-domain simulations of several CT $\Sigma \Delta \mathrm{Ms}$ intended for VDSL application.

\section{REFERENCES}

[1] J.A. Cherry and W.M. Snelgrove: Continuous-Time Delta-Sigma Modulators for High-Speed A/D Conversion. Kluwer, 2000.

[2] L. Breems and J.H. Huijsing: Continuous-Time Sigma-Delta Modulation for A/D Conversion in Radio Receivers. Kluwer, 2001.

[3] E. J. van der Zwan: "A 0.2-mW CMOS $\Sigma \Delta$ Modulator for Speech Coding with $80 \mathrm{~dB}$ Dynamic Range". IEEE Journal of Solid-State Circuits, Vol. 31, pp. 1873-1880, December 1996.

[4] O. Oliaei, H. Aboushady: "Jitter Effects in Continuous-Time $\Sigma \Delta$ Modulators with Delayed Return-to-Zero Feedback", Proc. of the 1998 IEEE International Conference on Electronics, Circuits and Systems, Vol.1, pp. 351-354.

[5] H. Tao, L. Toth, J. M. Khoury: "Analysis of Timing Jitter in Bandpass Sigma-Delta Modulators". IEEE Transactions on Circuits and Systems-II: Analog and Digital Signal Processing, Vol 46, pp. 991-1001, August 1999

[6] O. Oliaei, H. Aboushady: "State-Space Analysis of Clock Jitter in Continuous-Time Oversampling Data Converters". IEEE Transactions on Circuits and Systems-II: Analog Digital Signal Processing, Vol. 50, pp. 31-37, January 2003.

[7] L. Hernández, A. Wiesbauer, S. Patón and A. Di Giandomenico: "Modelling and Optimization of Low-Pass Continuous-Time Sigma-Delta Modulators for Clock Jitter Noise Reduction". Proc. of the 2004 International Symposium on Circuits and Systems, Vol. 1, pp. 1072-1075.

[8] M. Moyal, M. Groepl, H. Werker, G. Mitteregger, J. Schambacher: "A 700/900mW/Channel CMOS Dual Analog Front-End IC for VDSL with Integrated 11.5/14.5dBm Line Drivers". Proc. of the 2003 IEEE Int. Solid-State Circuits Conf., pp. 416-417.

[9] S. Yan, E. Sánchez-Sinencio: "A Continuous-Time $\Sigma \Delta$ Modulator With 88-dB Dynamic Range and 1.1-MHz Signal Bandwidth". IEEE Journal of Solid-State Circuits, Vol. 39, pp. 75-86, January 2004.

$\dagger 4$. Expression (15) is replaced in Fig.6 with the ideal value of $S N R$ for $\sigma_{\Delta T} \ll 1$. (a)

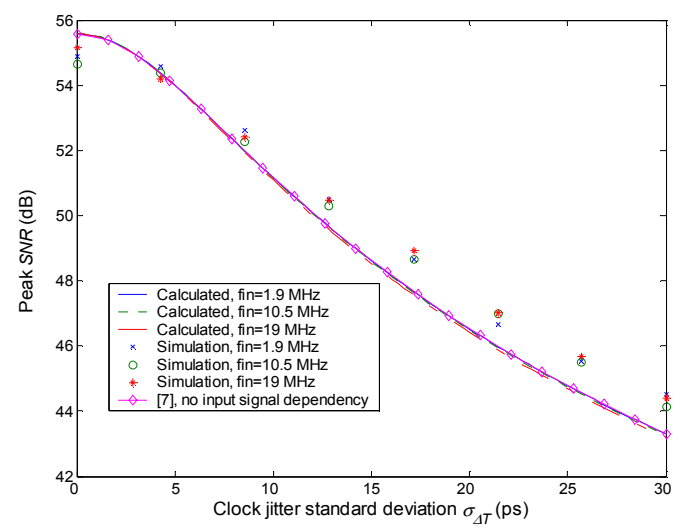

(b)

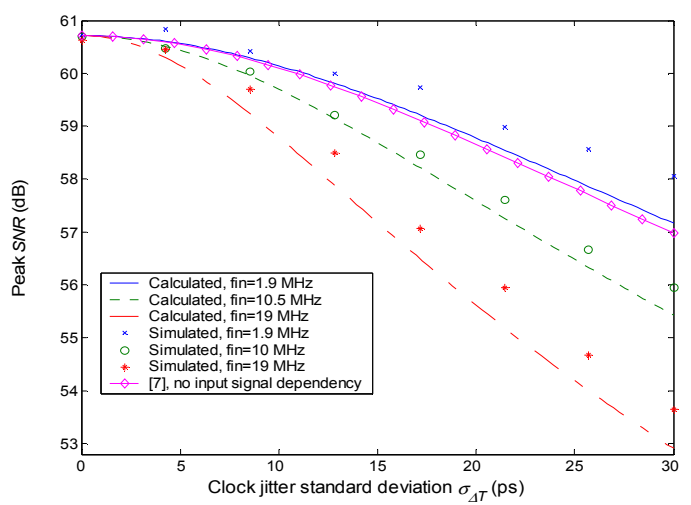

(c)

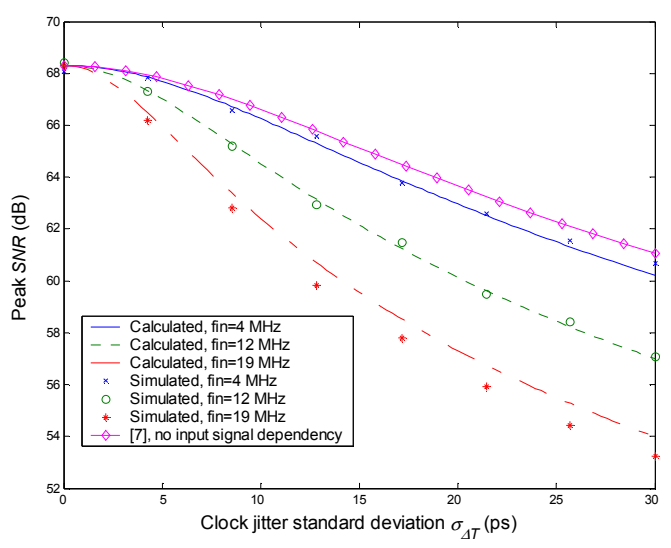

Figure 6. $S N R$ vs. $\sigma_{\Delta T}$ for different values of $f_{i}$ : (a) CT $\Sigma \Delta \mathrm{M} 1$. (b) CT $\Sigma \Delta \mathrm{M} 2$. (c) CT $\Sigma \Delta \mathrm{M} 3$.

[10]S. Patón, A. Di Giandoménico, L. Hernández, A. Wiesbauer, T. Pötscher and M. Clara: "A 70-mW 300-MHz CMOS Continuous-Time $\Sigma \Delta$ ADC With 15-MHz Bandwidth and 11 Bits of Resolution". IEEE Journal of Solid-State Circuits, Vol. 39, pp. 1056-1063, July 2004.

[11]R. Scherier and B. Zhang: " $\Delta \Sigma$ Modulators Employing Continuous-Time Circuitry". IEEE Transactions on Circuits and Systems-II: Analog and Digital Signal Processing, Vol 43, pp. 324-332, April 1996.

[12]J. G. Proakis, D. G. Manolakis: Digital Signal Processing. Principles, Algorithms and Appications. Prentice Hall, 1998

[13]J. Ruiz-Amaya, J.M. de la Rosa, F. Medeiro, F.V. Fernández, R. del Río, B. Pérez-Verdú and A. Rodríguez-Vázquez: "An Optimization-based Tool for the High-Level Synthesis of Discrete-time and Continuous-Time $\Sigma \Delta$ Modulators in the MATLAB/SIMULINK Environment". Proc. IEEE Int. Symp. Circuits and Systems, Vol V., pp. 97-100, 2004.

[14]R. Schreier, "An Empirical Study of High-Order Single-Bit Delta-Sigma Modulators". IEEE Transactions on Circuits and Systems-II: Analog and Digital Signal Processing, Vol 40, August 1993. 